Research Paper

\title{
Treatments of Sexual Dysfunction in Opioid Substitution Therapy Patients: A Systematic Review and Meta-Analysis
}

\author{
Fitri Fareez Ramli1 ${ }^{\bowtie}$, Muhammad Hasif Azizi² and Syed Alhafiz Syed Hashim ${ }^{1}$ \\ 1. Department of Pharmacology, Faculty of Medicine, Universiti Kebangsaan Malaysia, 56000 Cheras, Kuala Lumpur, Malaysia. \\ 2. Unit of Urology, Department of Surgery, Faculty of Medicine, Universiti Kebangsaan Malaysia, 56000 Cheras, Kuala Lumpur, Malaysia. \\ $\triangle$ Corresponding author: Fitri Fareez Ramli, Department of Pharmacology, Faculty of Medicine, Universiti Kebangsaan Malaysia, 56000 Cheras, Kuala Lumpur, \\ Malaysia. Tel: +603-9145 9545; Fax: 03-91459547; E-mail: fitrifareez@ppukm.ukm.edu.my.
}

(1) The author(s). This is an open access article distributed under the terms of the Creative Commons Attribution License (https://creativecommons.org/licenses/by/4.0/). See http://ivyspring.com/terms for full terms and conditions.

Received: 2020.12.28; Accepted: 2021.03.30; Published: 2021.04.12

\begin{abstract}
Sexual dysfunction is a common condition in the opioid substitution therapy (OST) population. We aimed to determine the efficacy and safety of treatment for sexual dysfunction in the OST population. We searched for interventional studies from Medline, PubMed, and Scopus. Three independent authors conducted a risk-of-bias assessment (RoB 2). A total of seven studies (five randomized-controlled trials, two quasi-experimental), including 473 patients with sexual dysfunction, were identified. Among these, three bupropion $(n=207)$, one trazodone $(n=75)$, two rosa Damascena $(n=100)$, and one ginseng $(n=91)$ studies had reported significantly improve various sexual functioning domains in both genders. In a meta-analysis, bupropion significantly increased male sexual function with standardized mean difference of $0.53 ; 95 \%$ confidence interval of $0.19-0.88 ; \mathrm{P}<0.01 ; \mathrm{I}^{2}=0$. The adverse effects were minor for all agents, and no significant difference between treatment and placebo groups in randomized-controlled trials. These agents have a promising future as therapy for sexual dysfunction in the OST population. However, given the limited sample size and number of studies, further studies should be conducted to confirm the use of these agents.
\end{abstract}

Key words: bupropion; ginseng; methadone; opioids; rosa Damascena; sexual dysfunction; trazodone

\section{Introduction}

Sexual function is one of the crucial aspects of life. Although it is not a life-threatening condition, deterioration in sexual function may reduce the sexual and overall quality of life in both patients and their partners [1]. Sexual problems are a broad spectrum of disorders, ranging from varying degrees of impairment of physiological components, often accompanied by psychological involvement [2]. Inadequate psychophysiological response in any phase, such as desire, arousal, orgasm, or resolution, may lead to reduced libido, excitement, vaginal lubrication, or penile erection; inability to reach orgasm; overall or sexual dissatisfaction; or pain [3].

In the opioid substitution therapy (OST) population, sexual dysfunction is the commonest condition reported in numerous pharmacovigilance studies. The prevalence of sexual dysfunction varies across the population, ranging from as low as $14 \%$ to as high as $93 \%$ in men on methadone maintenance treatment (MMT) [4-8]. In contrast, the data in women on MMT is limited, but the available report indicates a prevalence of 56.6\% [9]. Hypoactive sexual desire disorder in men and arousal impairment in women are the common sexual dysfunction in the MMT population. Moreover, the impairments are significantly higher in the MMT population compared to patients on buprenorphine maintenance treatment (BMT), another OST alternative $[9,10]$. On the contrary, the most common sexual dysfunctions in the general population are hypoactive sexual desire disorder and premature ejaculation in women and men, respectively [3].

Suppressive effects of the opioid on the hypothalamus-pituitary-gonadal axis are among the mechanisms of sexual dysfunction development in the OST population, particularly for potent opioid agonists such as methadone [11]. The mechanism has been proven in animal studies [11]. Various human 
studies also reported the association between hypogonadism and sexual dysfunction in patients receiving opioids [12]. This mechanism is not limited to men but may also contribute to the pathophysiology of sexual dysfunction in women [13, 14]. However, given the nature of the studies conducted, the established mechanism of sexual dysfunction in humans remained inconclusive [15]. Moreover, sexual dysfunction in the OST population may be more complicated, as the interaction between psychological, pharmacological, and social factors may worsen the sexual dysfunction condition $[6,7$, 16].

The discovery of sildenafil, a phosphodiesterase-5 (PDE5) inhibitor in 1998, has led to the development and expansion of PDE5 inhibitors class with improved pharmacological and psychological effects [17]. In fact, this is the first-line therapy for erectile dysfunction (ED). Other pharmacological interventions include testosterone replacement therapy and alprostadil. The management of sexual dysfunction in men is well-established. Various international and national guidelines are available to guide the clinician in managing patients with sexual dysfunction. These guidelines are not limited to ED but include other sexual dysfunctions, such as premature ejaculation [18-20]. The advancement of sexual dysfunction management in women occur in later years but has received wide attention over the past decade [21]. Similarly, some guidelines are available to guide various female sexual dysfunctions management, such as low desire, low arousal, orgasmic dysfunction, and sexual pain [21].

In contrast, the management of sexual dysfunction in the OST population is still limited and not widely studied. Lack of standardized management of sexual dysfunction in the OST population might be attributed to the lack of awareness and knowledge regarding sexual dysfunction among OST patients [6, 11]. A study conducted in methadone clinics that evaluated ED-related health-seeking behaviour reported almost half of the patients who were aware of having ED did not seek any treatment. Moreover, seeking a physician for help was the least popular treatment option in those who sought the treatment [6].

Due to the difference in terms of the pathophysiology of sexual dysfunction, the treatment option of sexual dysfunction in the OST population is different from the other population. Antidepressants such as bupropion and trazodone and herbal alternatives such as rosa Damascena and ginseng have been reported to significantly improve OST patients with sexual dysfunction [8, 22-27]. Although various clinical studies and reviews have explored the role of intervention for sexual dysfunction in many conditions, such as diabetes, stroke, and cancer population, the effectiveness and safety of sexual dysfunction intervention in the OST population has not been studied thoroughly [28-30]. We aimed to evaluate the effectiveness and safety profile of the treatments for sexual dysfunction from interventional studies in the OST population.

\section{Materials and Methods}

We adhered to the Preferred Reporting Items for Systematic Reviews and Meta-Analyses (PRISMA) guideline in preparing the review and article, except we did not register our protocol in any database [31].

\section{Literature Search}

Articles from three databases, Medline (via EBSCOhost - Medical Database), Scopus, and PubMed, were searched using keywords of (methadone or buprenorphine OR opioid) AND (sexual dysfunction OR erectile dysfunction OR hypoactive sexual desire disorder) from inception until November 2020 by two investigators (F.F.R. and S.A.S.H.) independently and any disagreement was resolved by discussion (Figure 1). Title screening was initially done, followed by abstract and full-text screening based on inclusion and exclusion criteria.

\section{Eligibility Criteria}

\section{Inclusion Criteria}

- Study designs: All interventional trials, including randomized-controlled trials (RCTs) and quasi-experimental;

- Participants: Studies in male and female patients aged $\geq 18$ years with sexual dysfunction of any severity on opioid substitution therapy (methadone or buprenorphine) for opioid use disorder;

- Interventions: Any pharmacological intervention, including herbal preparation as treatment. The comparator of active intention included placebo or other active pharmacological intervention;

- Outcomes: Studies with any mean score of validated questionnaires for sexual function assessment such as Arizona Sexual Experience Scales (ASEX) [25], Brief Sexual Function Inventory [22, 23], Clinical Global Impression for Sexual Function (CGI-SF) [27], Erectile Dysfunction Intensity Scale $[8,26]$, Female Sexual Function Index [22, 24], International Index of Erectile Function with 15-item [22, 23, 27], and Sexual Desire Inventory -2 [27]. Any minor and major adverse effects related to the interventions and the number of participants 
who dropped out and adverse effects were also retrieved;

- Data accessibility: Studies that were published as full papers and in English.

\section{Exclusion Criteria}

- Study designs: Any observational, animal study, and review articles;

- Participants: Patients on an opioid for pain management and sexual dysfunction secondary to organic causes;

- Interventions: Non-pharmacological intervention such as sex therapy or switching to another OST;

- Data accessibility: Studies published in languages other than English.

\section{Data Extraction}

The data from the included articles were extracted; first author's name, year of publication, country, population, number of participants, study design, age, gender, method (inclusion and exclusion criteria), duration and dose of OST, intervention (type, dose, route of administration, frequency, duration), and outcomes (sexual function and adverse effects). Any disagreement about the eligibility of the studies was resolved by discussion. For feasibility reason, authors were not contacted for missing data.

\section{Risk-of-bias Assessment}

The risk-of-bias assessment was conducted by three independent investigators (F.F.R., S.A.S.H., M.H.A.) using a revised tool for risk-of-bias (RoB 2) assessment for RCTs. Any disagreement was resolved by discussions [32]. Briefly, RoB 2 provides a risk-ofbias assessment in five domains; randomization process, deviation from the intended interventions, missing outcome data, measurement of the outcome, and selection of reported results [32]. Assessors are guided by signalling questions and domain-specific algorithms. There are three possible judgements by the authors that include "low risk of bias", "some concerns", or "high risk of bias" [32].

\section{Data Synthesis and Analysis}

Treatment effects were measured if there were at least two included studies to compare. The treatment effects were calculated using standardized mean differences (SMD) with a 95\% confidence interval (CI), given the difference in terms of tools used to measure sexual function. Forest plot inspection, standard

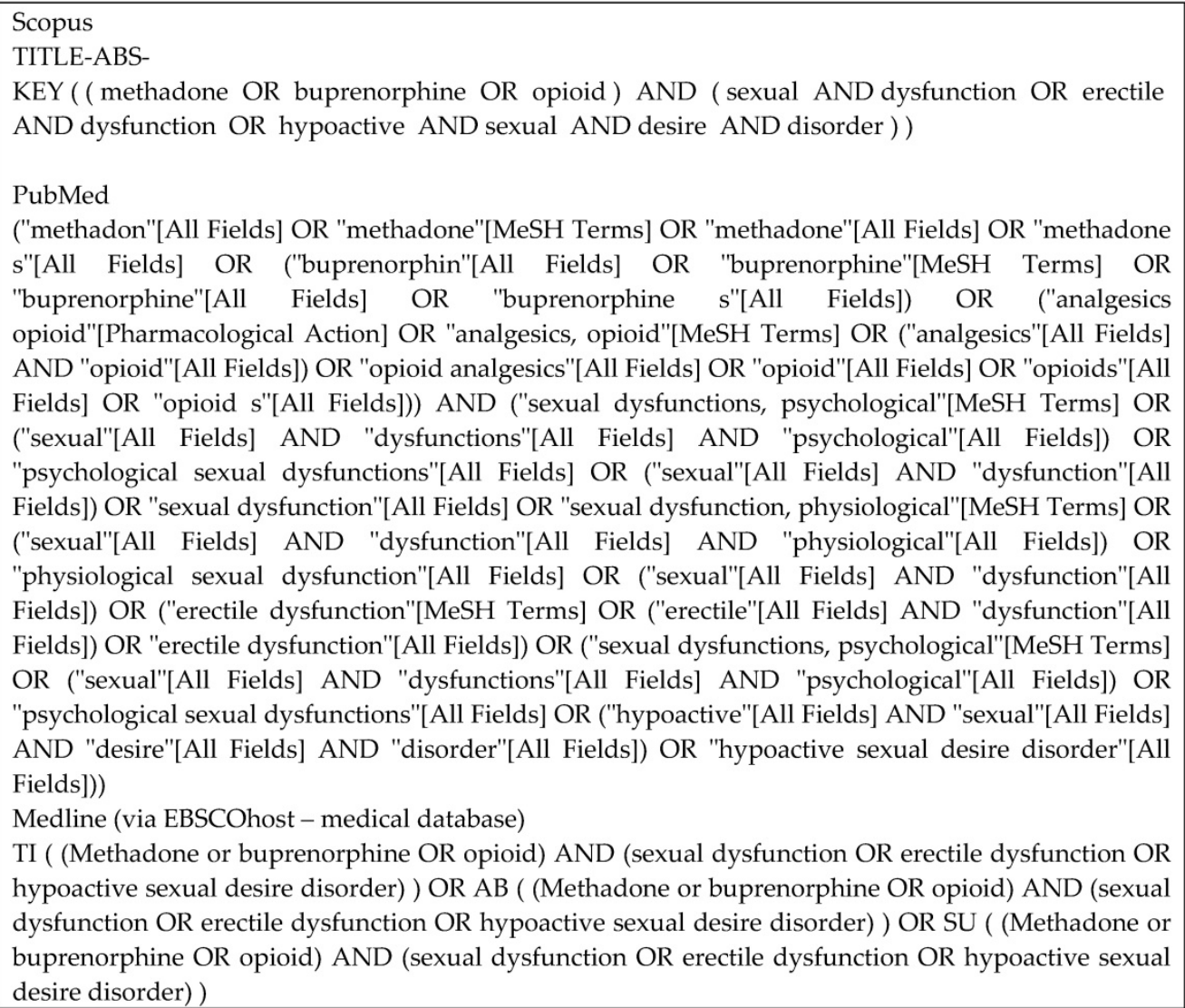


chi-square ( $p$-value $=0.1)$, and $I^{2}$ statistics were used to assess heterogeneity of meta-analysis in the case of no substantial clinical and methodological heterogeneity detected [33-35]. The $p$-value and $\mathrm{I}^{2}$ statistics determined the effects model used. Our study utilized the random-effects model as heterogeneity existed in terms of doses and the treatment's duration. We used Review Manager (RevMan) [Mac], Version 5.4, the Cochrane Collaboration, 2020.

\section{Results}

\section{Description of paper selection process}

A total of 632 articles were retrieved from three databases (Figure 2). After the screening of the title, 616 articles were removed, leaving 16 articles behind. All articles from the three databases were merged, and nine articles were removed. A final seven articles were included in our study after screening based on abstracts and full texts.

\section{Characteristics of included studies}

The majority of the studies were conducted in Iran, and only one study was conducted in Malaysia (Table 1). All studies were conducted in the MMT population with a sample size range of 50-91. Five studies were RCTs and two quasi-experimental studies. Most of the study population consisted of the male population. Only two studies had reported treatment for sexual dysfunction in the female population. All RCTs required the MMT duration of at least six months, and sexual dysfunction developed after MMT treatment. Two quasi-experimental studies had set the minimum duration of MMT at 30 days [8, 26]. Other eligibility criteria included lack of the following conditions; other psychiatric comorbidities or conditions [10, 22-25], other substance use disorder $[23,24,36]$, obvious organic illnesses $[8,26,27,36]$, not on other psychotropic drugs [8, 22-24, 27, 36], anti-viral treatment [8, 24, 27], and sexual enhancement therapy $[8,24,27]$, somatic problems [22], current marital issues [22], non-adherence to the protocol [24], allergy [22], intention to become pregnant, pregnancy, or lactation [24], and adverse effects related to treatment [24].

\section{Treatments for sexual dysfunction}

Three studies conducted using bupropion with a dose of $100-300 \mathrm{mg}$ for 6-8 weeks (Table 2). Two studies conducted using Rosa Damascena in the male and female population. Another study used ginseng or trazodone.

\section{Risk-of-bias assessment}

Risk-of-bias assessment for all RCT studies demonstrated all, but one had a low risk of bias for the randomization process (Table 3). Only Salehi, Barekatain [36] had an unclear risk of bias for the randomization process domain. Four out of five studies had a high risk of bias in the selection of the reported result domains.

Table 1. Characteristics of included studies

\begin{tabular}{|c|c|c|c|c|c|c|c|}
\hline $\begin{array}{l}\text { Author's } \\
\text { name }\end{array}$ & Year & Country & $\begin{array}{l}\text { Study } \\
\text { population }\end{array}$ & $\mathrm{n}$ & Design & Gender (\%) & Age (years) \\
\hline Tatari [26] & 2010 & Iran & MMT & 75 & Quasi & Men (100\%) & $39.5^{\dagger}\left(18-51^{\ddagger}\right)$ \\
\hline Tatari [8] & 2014 & Iran & MMT & 67 & Quasi & Men (100\%) & $36.2^{\dagger}\left(21-53^{\ddagger}\right)$ \\
\hline Salehi [25] & 2015 & Iran & MMT & 60 & RCT & Men (100\%) & $\begin{array}{l}\text { P:43.1†; T: } \\
\left.41.5^{\dagger}\right)\end{array}$ \\
\hline $\begin{array}{l}\text { Farnia } \\
{[23]}\end{array}$ & 2017 & Iran & MMT & 50 & RCT & Men (100\%) & $40.0^{\dagger}$ \\
\hline $\begin{array}{l}\text { Farnia } \\
{[24]}\end{array}$ & 2017 & Iran & MMT & 50 & $\mathrm{RCT}$ & $\begin{array}{l}\text { Women } \\
(100 \%)\end{array}$ & $38.8^{\dagger}$ \\
\hline Yee [27] & 2018 & Malaysia & MMT & 80 & RCT & Men $(100 \%)$ & $42.8^{\dagger}(25-60 \ddagger)$ \\
\hline $\begin{array}{l}\text { Farnia } \\
{[22]}\end{array}$ & 2019 & Iran & MMT & 91 & RCT & $\begin{array}{l}\text { Men } \\
(64.9 \%), \\
\text { Women } \\
(35.1 \%)\end{array}$ & $\begin{array}{l}\text { W: } 39.0^{+} ; \mathrm{M}: \\
40.6^{\dagger}\end{array}$ \\
\hline
\end{tabular}

tmean; łrange. Abbreviations: M: men; MMT: methadone maintenance treatment; n: sample size; NA: not available; P: placebo group; RCT: randomized-controlled trial; T: treatment group; W: women.

Table 2. Types, route, dose and duration of treatment of sexual dysfunction in MMT population

\begin{tabular}{lllll}
\hline Ref & Agent & Route & Dose daily & Duration (week) \\
\hline$[26]$ & Trazodone & Oral & $100 \mathrm{mg}$ & 6 \\
{$[8]$} & Bupropion & Oral & $100 \mathrm{mg}$ & 6 \\
{$[25]$} & Bupropion & Oral & $200 \mathrm{mg}$ & 8 \\
{$[23]$} & Rosa Damascena & Oral & $2 \mathrm{ml}$ & 8 \\
{$[24]$} & Rosa Damascena & Oral & $2 \mathrm{ml}$ & 8 \\
{$[27]$} & Bupropion & Oral & $300 \mathrm{mg}$ & 6 \\
{$[22]$} & Ginseng & Oral $^{*}$ & $1000 \mathrm{mg}$ & 4 \\
\hline
\end{tabular}

*Each capsule contained $250 \mathrm{mg}$ dry powder of ginseng radix. Abbreviations: MMT: methadone maintenance treatment; Ref: reference.

\section{The effects of treatment on sexual function}

The majority of the studies had reported improvement in a variety of sexual function domains (Table 4). Both rosa Damascena and ginseng were reported to improve sexual drive, erection, ejaculation, problem assessment, sexual satisfaction, and overall sexual function in the male MMT population. On the other hand, bupropion was reported to improve erectile function, sexual desire, dyadic sexual desire, intercourse satisfaction, and overall sexual function in one RCT study. In contrast, another study reported a trend towards sexual function improvement but non-significant results in terms of overall sexual function.

In the female MMT population, both ginseng and rosa Damascena were reported to improve desire, arousal, orgasm, pain, and overall sexual function (Table 5). However, the mixed results were reported in terms of lubrication and satisfaction between these two treatments. 
Table 3. Risk-of-bias assessment for included randomized-controlled trials

\begin{tabular}{|c|c|c|c|c|c|c|}
\hline Reference & Randomization process & $\begin{array}{l}\text { Deviations from intended } \\
\text { intervention }\end{array}$ & Missing outcome data & $\begin{array}{l}\text { Measurement of the } \\
\text { outcome }\end{array}$ & $\begin{array}{l}\text { Selection of the } \\
\text { reported results }\end{array}$ & Overall risk \\
\hline [25] & $?$ & + & + & + & - & - \\
\hline [23] & + & + & + & + & - & - \\
\hline [24] & + & + & + & + & + & + \\
\hline [27] & + & + & + & + & - & - \\
\hline [22] & + & + & - & + & - & - \\
\hline
\end{tabular}

+ low risk-of-bias; ? some concern; - high risk-of-bias.

Table 4. Effects of treatment on male sexual functioning in the MMT population

\begin{tabular}{|c|c|c|c|c|c|c|c|c|c|c|c|c|c|c|c|c|c|c|c|}
\hline Tool & & ASEX & BSFI & & & & & & CGI-SF & EDIS & IIEF & & & & & & SDI-2 & & \\
\hline Ref & Agent & Total & $\begin{array}{l}\text { Sexual } \\
\text { drive }\end{array}$ & Erection & Ejaculation & $\begin{array}{l}\text { Problem } \\
\text { assessment }\end{array}$ & $\begin{array}{l}\text { Sexual } \\
\text { satisfaction }\end{array}$ & Total & Total & Total & Erectile & Orgasmic & SD & $\begin{array}{l}\text { Intercourse } \\
\text { satisfaction }\end{array}$ & $\begin{array}{l}\text { Overall } \\
\text { satisfaction }\end{array}$ & Total & DSD & SSD & Total \\
\hline [26] & Trazodone & & & & & & & & & $\uparrow^{*}$ & & & & & & & & & \\
\hline [8] & Bupropion & & & & & & & & & $\uparrow^{*}$ & & & & & & & & & \\
\hline [25] & Bupropion & NS & & & & & & & & & & & & & & & & & \\
\hline [23] & $\begin{array}{l}\text { Rosa } \\
\text { Damascena }\end{array}$ & & $\uparrow^{\ddagger}$ & $\uparrow^{\ddagger}$ & $\uparrow^{\ddagger}$ & $\uparrow^{\ddagger}$ & $\uparrow^{* \neq}$ & $\uparrow^{\ddagger}$ & & & & & & & & $\uparrow^{\ddagger}$ & & & \\
\hline [27] & Bupropion & & & & & & & & $\uparrow^{*+}$ & & $\uparrow^{*} \dagger$ & NS & $\uparrow^{\dagger}$ & $\uparrow^{*}$ & NS & $\uparrow^{\dagger}$ & $\uparrow^{*}+$ & NS & $\uparrow^{\dagger}$ \\
\hline [22] & Ginseng & & $\uparrow \neq$ & $\uparrow \neq$ & $\uparrow^{\ddagger}$ & $\uparrow \neq$ & $\uparrow^{*} \neq$ & $\uparrow \neq$ & & & & & & & & $\uparrow^{* *}$ & & & \\
\hline
\end{tabular}

*Treatment vs placebo (at the end of the treatment); twithin treatment group (baseline vs week 2/4/6); $¥$ Time x Group interaction.

ASEX: Arizona sexual experience scale; BSFI: Brief Sexual Function Inventory; CGI-SF: Clinical Global Impression Scale adapted for Sexual Function; DSD: dyadic sexual desire; EDIS: erectile dysfunction intensity scale; EF: erectile functioning; IIEF: International Index of Erectile Function; MMT: methadone maintenance treatment; SD: sexual desire; SDI: Sexual desire inventory -2 (Malay version); SF: sexual function; SSD: solitary sexual desire.

\section{Meta-analysis of bupropion effect on sexual function}

Only two RCT studies on bupropion as a treatment of sexual dysfunction were eligible for meta-analysis. The mean and standard deviation of the total ASEX score [36] and CGI-SF [27] were included in the analysis to calculate SMD. A meta-analysis of two RCT studies with a total of 132

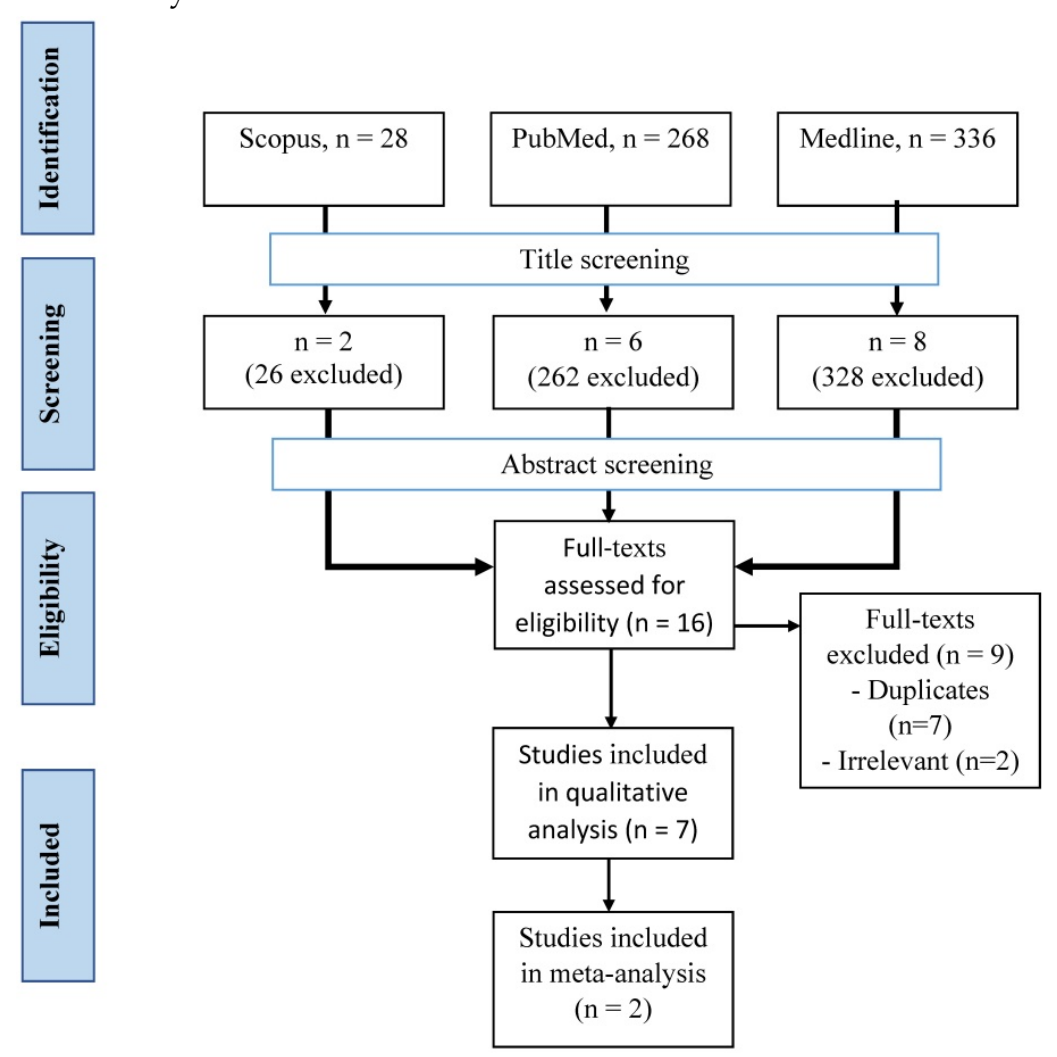

Figure 2. Selection process of the articles. patients demonstrated a significant effect of bupropion in the treatment of sexual dysfunction with a SMD of 0.53 ; $95 \%$ confidence interval of $0.19-0.88$; $\mathrm{P}$ $<0.01 ; \mathrm{I}^{2}=0$ (Figure 3).

\section{Dropout rate and adverse effects of the treatments}

The total dropout rate ranged from $0-36.3 \%$ in four studies. The reasons for dropout included no reason, adverse reactions (AEs), no sexual partner, not effective, and other reasons. No studies reported any major AE. Only mild AEs were reported, including insomnia, inability to concentrate, and rashes in patients receiving bupropion. One-third of patients receiving trazodone had reported sedation. In patients receiving ginseng, AEs included agitation, sleeplessness, high blood pressure, stomach ache, diarrhoea, and rash. No AEs were reported and mentioned in patients receiving rosa Damascena.

\section{Discussion}

Our systematic review found four potential pharmacological agents; bupropion, trazodone, rosa Damascena, and ginseng [8, 22-27]. Most of the studies were conducted in the male MMT population [8, 22, 23, 25-27]. The male predominance might be attributed to a low number of studies in the female MMT 
population and a higher proportion of men in this population [8, 22, 23, 25-27]. In risk-of-bias assessments for RCTs, most of the studies had high risk-of-bias in reporting [22, 23, 25, 27]. The majority of these studies had not specified certain measurement tools in the registered protocol and selective outcome measurement and analysis [22, 23, $25,27]$.

Table 5. Effects of treatment on female sexual functioning in the MMT population

\begin{tabular}{|c|c|c|c|c|c|c|c|}
\hline Tools & FSFI & & & & & & \\
\hline Ref & Desire & Arousal & Lubrication & Orgasm & Satisfaction & Pain & Total \\
\hline [24] & $\uparrow^{* \neq}$ & $\uparrow^{* \neq}$ & $\uparrow \ddagger$ & $\uparrow \ddagger$ & $\uparrow \ddagger$ & $\uparrow \ddagger$ & $\uparrow^{* \neq}$ \\
\hline [22] & $\uparrow \ddagger$ & $\uparrow \ddagger$ & NS & $\uparrow^{* \neq}$ & NS & $\uparrow \ddagger$ & $\uparrow \ddagger$ \\
\hline
\end{tabular}

FSFI: Female Sexual Function Inventory; MMT: methadone maintenance treatment; NS: not significant;

${ }^{*}$ Treatment vs placebo; ${ }^{\dagger}$ within treatment group (baseline vs week $2 / 4 / 6$ ); ${ }^{\text {}}$ Time $x$ Group interaction; $\uparrow:$ improved.

Table 6. Dropout rate, reason and adverse effects of patients on treatment of sexual dysfunction

\begin{tabular}{|c|c|c|c|c|c|c|}
\hline \multirow[t]{2}{*}{ Ref } & \multirow[t]{2}{*}{$\mathrm{n}$} & \multicolumn{3}{|c|}{ Dropout } & \multirow[t]{2}{*}{ Reason } & \multirow[t]{2}{*}{ Adverse effects } \\
\hline & & Tx & Placebo & Total & & \\
\hline [26] & 75 & 15 & - & $15(20 \%)$ & $\begin{array}{l}33.3 \% \text { due to } \\
\text { sedation. }\end{array}$ & Sedation \\
\hline [8] & 67 & 15 & - & $15(22.4 \%)$ & Not specified & $\begin{array}{l}\text { No major side } \\
\text { effects. }\end{array}$ \\
\hline [25] & 60 & Nil & Nil & Nil & Nil & Not reported \\
\hline [23] & 50 & Nil & Nil & Nil & Nil & Not reported \\
\hline [24] & 50 & Nil & Nil & Nil & Nil & Not reported \\
\hline [27] & 80 & 14 & 15 & $29(36.3 \%)$ & $\begin{array}{l}\text { No reason: } 4 \text { vs } 5^{\dagger} \\
\text { AE: } 9 \text { vs } 5^{\dagger} \\
\text { No sexual partner: } \\
1 \text { vs } 1^{\dagger} \\
\text { Not effective: } 0 \text { vs } \\
3^{\dagger} \\
\text { VA: } 0 \text { vs } 1^{\dagger} \\
\text { EPTB: } 1 \text { vs } 0^{\dagger}\end{array}$ & $\begin{array}{l}\text { No significant } \\
\text { different between } \\
\text { groups. } \\
\text { AEs in treatment } \\
\text { groups: insomnia, } \\
\text { skin itchiness, } \\
\text { cannot concentrate, } \\
\text { constipation. }\end{array}$ \\
\hline \multirow[t]{2}{*}{ [22] } & M:54 & 4 & 2 & $6(11.1 \%)$ & $\begin{array}{l}\text { AE: } 4 \text { vs } 0^{\dagger} \\
\text { NC: } 0 \text { vs } 4^{\dagger}\end{array}$ & \multirow{2}{*}{$\begin{array}{l}\text { Sleeplessness, } \\
\text { agitation, high BP, } \\
\text { stomach ache, } \\
\text { diarrhoea, rash. }\end{array}$} \\
\hline & W:37 & 6 & 5 & $11(29.7 \%)$ & $\begin{array}{l}\text { AE: } 5 \text { vs } 3^{+} ; \\
\text {NC: } 1 \text { vs } 2^{+}\end{array}$ & \\
\hline
\end{tabular}

†Treatment vs placebo; AE: adverse effect; BP: blood pressure; EPTB: extrapulmonary tuberculosis; -: no control group; W: women; M: men; NC: non-compliance; Ref: reference; Tx: treatment; VA: vehicle accident.

We were unable to proceed with meta-analysis for trazodone [26] and ginseng [22] because only one study was found in our systematic review. In terms of rosa Damascena, two studies included were not homogenous as one was conducted in the male MMT population, while another in the female MMT population. Given the differences in gender and sexual functioning, we did not proceed with meta-analysis. Furthermore, another reason is the quasi-experimental nature of trazodone [26] and bupropion [8] studies. In contrast, we found two studies on bupropion that were eligible for further analysis $[25,27]$.

We found that bupropion significantly improved overall sexual functioning, sexual desire, erectile function, and intercourse satisfaction in men $[8,27$, 36]. Both quasi-experimental and RCT had reported positive impacts in numerous domains (sexual desire, erectile function, intercourse satisfaction) of sexual function. However, the effects of bupropion on other domains were not significantly improved (orgasmic function and overall satisfaction). Moreover, our meta-analysis had reported significant positive effects of bupropion on male sexual function. Our results agree with another RCT study conducted in the male population on selective serotonin reuptake inhibitor (SSRI) treatment for depression [37]. The underlying mechanism of bupropion is related to its non-typical mechanism of action via inhibition of noradrenaline and dopamine reuptake without compromising serotonin regulation. Serotonin enhancement is related to sexual dysfunction in patients on SSRIs [38]. The dual-action of bupropion on depression and sexual dysfunction at a higher therapeutic dose has the advantage for the use in the MMT population, in which at least half of the population is affected by depression [39]. Other than that, increased testosterone is another potential mechanism of improved sexual function in MMT patients on bupropion treatment [27].

In terms of adverse reaction, no major or significant difference was found between treatment (bupropion) and placebo groups $[8,27,36]$. Yee, Loh [27] reported that insomnia was the highest adverse effect, affecting approximately one-tenth of the study population. Similarly, Safarinejad [37] reported that no major AEs occurred in patients with SSRI-induced sexual dysfunction patients. However, minor AEs related to neurological, musculoskeletal, respiratory, and gastrointestinal were reported to be significantly higher in patients receiving bupropion [37]. These AEs might be attributed to the pharmacological effect of bupropion on the enhancement of the functions and blockades of dopamine and noradrenaline receptors [38].

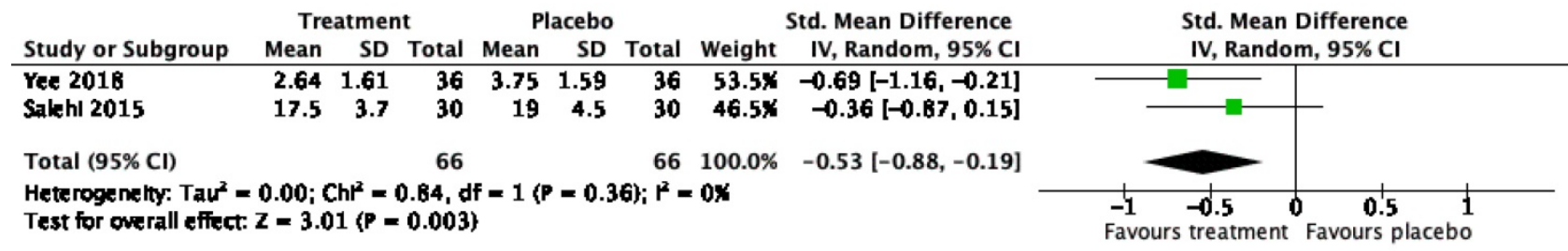

Figure 3. Forest plot on effect of bupropion on male sexual dysfunction. 
Trazodone is another potential pharmacological agent for the treatment of sexual dysfunction in men. Tatari, Farnia [26] had conducted a study to determine the effects of trazodone in 75 MMT patients with ED. Despite a significant improvement, the report is limited in terms of which specific domain of erectile function was improved. A meta-analysis study of trazodone effects on erectile function reported a significant improvement in patients with psychogenic cause, but not for physiological and mixed-related ED [40]. Interestingly, significant improvement in the MMT population was achieved at a low dose (50 mg/day) [26], a dose that exerts hypnotic and neuroprotective effects and not anti-depressant effect [41]. The mechanism of action of trazodone is attributed to its high affinity and blockade of alpha-1 adrenoceptor in penile tissue [42]. Concerning adverse reaction, sedation was the only adverse effect reported, leading to dropping out of the patients [26]. A similar finding was reported in a systematic review conducted by Fink, MacDonald [40] in the general population.

Ginseng is an herbal alternative for the treatment of sexual function. Farnia, Alikhani [22] had reported significant improvement in numerous domains of sexual function in both genders. Similar effects were reported in other populations such as men and postmenopausal women [43, 44]. Sexual improvement by ginseng might be explained by the local and central effects of ginsenosides, the active components present in ginseng. Ginsenoside may cause vasodilation and relaxation of the corpus cavernosum, facilitating penile erection directly or via nitric oxide released from perivascular nerves or endothelial cells [45]. Moreover, ginseng causes sexual improvement via neurohormonal effects at hypothalamus and pituitary levels [45]. The mechanism of ginseng in women might be attributed to the ability of ginseng to enhance estrogen levels, promoting estrogenic changes on the female reproductive system, such as vaginal smooth muscle relaxation and increased vaginal blood flow [46, 47]. Adverse effects of ginseng reported were related to minor neurological, rash, and gastrointestinal symptoms. Similar findings were reported in other populations [44]. Other than that, Oh, Chae [44] reported vaginal bleeding in postmenopausal women treated with ginseng.

Another herbal medicine reported to have the potential for sexual dysfunction in the OST population is Rosa Damascena. Two studies had reported significant improvement in male and female patients on MMT. The underlying mechanism is not fully understood. Rosa Damascena's effects on the hypothalamus-pituitary-gonadal axis may partly explain its sexual enhancement effects. Farnia, Tatari
[24] reported significant elevations of estradiol and progesterone and reduction of prolactin in females receiving rosa Damascena. In the male MMT population, serum testosterone levels were significantly increased in the treatment group [23]. Similar findings were reported in male and female patients with SSRI-induced sexual dysfunction in the major depressive disorder [48, 49]. The preclinical study supports the positive effect in elevations of follicle-stimulating hormone, luteinizing hormone, and testosterone in male rats treated with rosa Damascena [50]. However, negligible correlations were observed between hormonal levels and sexual function in the male and female MMT population [23, 24].

Despite promising evidence of treatment for opioid-induced sexual dysfunction, these findings should be interpreted with caution due to the limited number of studies, small sample size, and strict exclusion criteria. Six out of seven studies were conducted in Iran. We postulated a high prevalence of patients with opioid misuse and the availability of natural resource that is native to Iran (Rosa Damascena) are the reasons why most of the published data on sexual treatment mainly came from Iran. In contrast, other regions in the world may have different treatment strategies, such as switching methadone with buprenorphine, an agent with a lower risk of sexual dysfunction [10]. These factors may limit the generalizability of the studies to other populations. Sexual dysfunction in OST patients is complex. Most of the studies in our report included opioid use disorder patients only on methadone without any comorbidity or the use of other prescribed or illicit drugs. Many patients on OST have a dual diagnosis or other comorbidities in the real-world situation, which requires other pharmacological intervention. Methadone has wide pharmacokinetic interindividual variability, which may be affected by drug-drug interaction, genetic mark-up and underlying disease [16]. Another limitation is the lack of adverse effects reporting in three RCTs, making it difficult to assess safety profile in meta-analysis. The result must be interpreted with caution, given the high risk-of-bias in most of the studies secondary to selective outcome reporting. Future research should emphasize on a larger sample size, more studies in other countries, adherence to registered protocol, and improved quality of reported articles to reduce the risk of bias. Recommendations for quality improvement would include an explanation for protocol deviation and a statement regarding the safety profile or adverse effect, even if no adverse effects were observed during the trials. 


\section{Conclusions}

Bupropion, ginseng, rosa Damascena and trazodone have a promising future as therapy for male sexual dysfunction, particularly in sexual desire, erection, ejaculation, problem assessment, and sexual satisfaction domains. In the female OST population, ginseng and rosa Damascena may have the potential to treat sexual dysfunction related to desire, arousal, lubrication, orgasm, satisfaction, and pain. However, given the limited sample size and number of studies, further studies should be conducted to confirm the efficacy and safety.

\section{Abbreviations}

AE: adverse effects; ASEX: Arizona Sexual Experience Scales; CGI-SF: Clinical Global Impression for Sexual Function; CI: confidence interval; ED: erectile dysfunction; MMT: methadone maintenance treatment; OST: opioid substitution therapy; PDE-5: phosphodiesterase-5; PRISMA: Preferred Reporting Items for Systematic Reviews and Meta-Analyses; RCT: randomized-controlled trial; RoB2: revised tool for risk of bias; SMD: standardized mean differences; SSRI: selective serotonin reuptake.

\section{Acknowledgements}

The researchers thank Universiti Kebangsaan Malaysia for providing Faculty of Medicine, Research Grant (FF-2020-512).

\section{Funding}

This research was funded by the Faculty of Medicine, Universiti Kebangsaan Malaysia, grant number FF-2020-512.

\section{Author Contributions}

Conceptualization, F.F.R.; methodology, F.F.R. and S.A.S.H.; formal analysis, F.F.R., S.A.S.H and M.H.A.; writing-original draft preparation, F.F.R; writing-review and editing, F.F.R., S.A.S.H and M.H.A. All authors have read and agreed to the published version of the manuscript.

\section{Competing Interests}

The authors have declared that no competing interest exists.

\section{References}

1. Abolfotouh M, Al Helali N. Effect of erectile dysfunction on quality of life. EMHJ-Eastern Mediterranean Health Journal. 2001; 7 (3): 510-518.

2. Shafer LC. Sexual Disorders or Sexual Dysfunction. Massachusetts General Hospital Handbook of General Hospital Psychiatry E-Book. 2017: 279.

3. Grover S, Shouan A. Assessment Scales for Sexual Disorders-A Review. J Psychosexual Health. 2020; 2: 121-38.

4. Jaafar NRN, Mislan N, Aziz SA, Baharudin A, Ibrahim N, Midin M, et al. Risk factors of erectile dysfunction in patients receiving methadone maintenance therapy. J Sex Med. 2013; 10: 2069-76.
5. Brown R, Balousek S, Mundt M, Fleming M. Methadone maintenance and male sexual dysfunction. J Addict Dis. 2005; 24: 91-106.

6. Ramli FF, Shuid AN, Pakri Mohamed RM, Tg Abu Bakar Sidik TMI, Naina Mohamed I. Health-Seeking Behavior for Erectile Dysfunction in Methadone Maintenance Treatment Patients. Int J Environ Res Public Health. 2019; 16: 4249 .

7. Ramli FF, Tg Abu Bakar Sidik TMI, Naina Mohamed I. Sexual Inactivity in Methadone Maintenance Treatment Patients. Int J Environ Res Public Health. 2020; 17: 1993

8. Tatari F, Shakeri J, Farnia V, Heidari F, Rezaei M. Bupropion in methadone induced erectile dysfunction. Ann Psychiatry Ment Health. 2014; 2: 1015.

9. Zamboni L, Franceschini A, Portoghese I, Morbioli L, Lugoboni F. Sexual Functioning and Opioid Maintenance Treatment in Women. Results from a Large Multicentre Study. Front Behav Neurosci. 2019; 13: 97.

10. Yee A, Loh HS, Hisham Hashim HM, Ng CG. The prevalence of sexual dysfunction among male patients on methadone and buprenorphine treatments: a meta-analysis study. J Sex Med. 2014; 11: 22-32.

11. Ramli F, Shuid A, Mohamed P, Mohamed R, Naina Mohamed I. Erectile dysfunction and methadone maintenance therapy. Med Health. 2020; 15: 5-17.

12. Bliesener N, Albrecht S, Schwager A, Weckbecker K, Lichtermann D, Klingmüller D. Plasma testosterone and sexual function in men receiving buprenorphine maintenance for opioid dependence. J Clin Endocrinol Metab. 2005; 90: 203-6.

13. Daniell HW. Opioid endocrinopathy in women consuming prescribed sustained-action opioids for control of nonmalignant pain. J Pain. 2008; 9: 28-36.

14. Rhodin A, Stridsberg M, Gordh T. Opioid endocrinopathy: a clinical problem in patients with chronic pain and long-term oral opioid treatment. Clin J Pain. 2010; 26: 374-80.

15. Hallinan R, Byrne A, Agho K, McMahon CG, Tynan P, Attia J. Hypogonadism in men receiving methadone and buprenorphine maintenance treatment. Int J Androl. 2009; 32: 131-9.

16. Ramli FF. Pharmacogenomics biomarkers for personalized methadone maintenance treatment: The mechanism and its potential use. Bosn J Basic Med Sci. 2021; 21: 145-54

17. Gong B, Ma M, Xie W, Yang X, Huang Y, Sun T, et al. Direct comparison of tadalafil with sildenafil for the treatment of erectile dysfunction: a systematic review and meta-analysis. Int Urol Nephrol. 2017; 49: 1731-40.

18. Hatzimouratidis K, Amar E, Eardley I, Giuliano F, Hatzichristou D, Montorsi $\mathrm{F}$, et al. Guidelines on male sexual dysfunction: erectile dysfunction and premature ejaculation. Eur Urol. 2010; 57: 804-14.

19. Hackett G, Kirby M, Wylie K, Heald A, Ossei-Gerning N, Edwards D, et al. British Society for Sexual Medicine guidelines on the management of erectile dysfunction in men - 2017. J Sex Med. 2018; 15: 430-57.

20. Bella AJ, Lee JC, Carrier S, Bénard F, Brock GB. 2015 CUA Practice guidelines for erectile dysfunction. Can Urol Assoc J. 2015; 9: 23.

21. Krakowsky Y, Grober ED. A practical guide to female sexual dysfunction: An evidence-based review for physicians in Canada. Can Urol Assoc J. 2018; 12: 211-6.

22. Farnia V, Alikhani M, Ebrahimi A, Golshani S, Sadeghi Bahmani D, Brand S. Ginseng treatment improves the sexual side effects of methadone maintenance treatment. Psychiatry Research. 2019; 276: 142-50.

23. Farnia V, Tatari F, Alikhani M, Shakeri J, Taghizadeh M, Karbasizadeh H, et al. Rosa Damascena oil improved sexual function and testosterone in male patients with opium use disorder under methadone maintenance therapy-results from a double-blind, randomized, placebo-controlled clinical trial. Drug Alcohol Depend. 2017; 176: 117-25.

24. Farnia V, Tatari F, Alikhani M, Yazdchi K, Taghizadeh M, Sadeghi Bahmani D, et al. Rosa Damascena oil improved methadone-related sexual dysfunction in females with opioid use disorder under methadone maintenance therapy - results from a double-blind, randomized, and placebo-controlled trial. J Psychiatr Res. 2017; 95: 260-8.

25. Salehi M, Barekatain M, Faghani F, Karimian N, Molaeinezhad M, Asadalloahi $\mathrm{G}$, et al. Bupropion efficacy on sexual dysfunction among male patients on methadone maintenance therapy: a double-blind placebo-controlled trial. Sex Relatsh Ther. 2015; 30: 364-75.

26. Tatari F, Farnia V, Nasiri RF, Najafi F. Trazodone in methandone induced erectile dysfunction. Iran J Psychiatry. 2010; 5: 164-6.

27. Yee A, Loh HS, Ong TA, Ng CG, Sulaiman AH. Randomized, Double-Blind, Parallel-Group, Placebo-Controlled Trial of Bupropion as Treatment for Methadone-Emergent Sexual Dysfunction in Men. Am J Mens Health. 2018; 12: $1705-18$.

28. Candy B, Jones L, Vickerstaff V, Tookman A, King M. Interventions for sexual dysfunction following treatments for cancer in women. Cochrane Database Syst Rev. 2016.

29. Stratton $\mathrm{H}$, Sansom J, Brown-Major A, Anderson P, Ng L. Interventions for sexual dysfunction following stroke. Cochrane Database Syst Rev. 2020.

30. Vardi M, Nini A. Phosphodiesterase inhibitors for erectile dysfunction in patients with diabetes mellitus. Cochrane Database Syst Rev. 2007; 2007: CD002187-CD.

31. Moher D, Liberati A, Tetzlaff J, Altman DG, Group P. Preferred reporting items for systematic reviews and meta-analyses: the PRISMA statement. PLoS Med. 2009; 6: e1000097.

32. Sterne JA, Savović J, Page MJ, Elbers RG, Blencowe NS, Boutron I, et al. RoB 2: a revised tool for assessing risk of bias in randomised trials. BMJ. 2019; 366. 
33. Higgins JP, Thompson SG, Deeks JJ, Altman DG. Measuring inconsistency in meta-analyses. BMJ. 2003; 327: 557-60.

34. Higgins JP, Thompson SG. Quantifying heterogeneity in a meta-analysis. Stat Med. 2002; 21: 1539-58

35. Deeks JJ, Higgins JP, Altman DG, Group CSM. Analysing data and undertaking meta-analyses. Cochrane handbook for systematic reviews of interventions. 2019; p: 241-84.

36. Salehi M, Barekatain M, Faghani F, Karimian N, Molaeinezhad M, Asadalloahi GA, et al. Bupropion efficacy on sexual dysfunction among male patients on methadone maintenance therapy: a double-blind placebo-controlled trial. Sexual \& Relationship Therapy. 2015; 30: 364-75.

37. Safarinejad MR. The effects of the adjunctive bupropion on male sexual dysfunction induced by a selective serotonin reuptake inhibitor: a doubleblind placebo-controlled and randomized study. BJU Int. 2010; 106: 840-7.

38. Stahl SM, Pradko JF, Haight BR, Modell JG, Rockett CB, Learned-Coughlin S. A review of the neuropharmacology of bupropion, a dual norepinephrine and dopamine reuptake inhibitor. Prim Care Companion J Clin Psychiatry. 2004; 6: 159.

39. Peles E, Schreiber S, Naumovsky Y, Adelson M. Depression in methadone maintenance treatment patients: rate and risk factors. J Affect Disord. 2007; 99: 213-20.

40. Fink H, MacDonald R, Rutks I, Wilt T. Trazodone for erectile dysfunction: a systematic review and meta-analysis. BJU Int. 2003; 92: 441-6.

41. Settimo L, Taylor D. Evaluating the dose-dependent mechanism of action of trazodone by estimation of occupancies for different brain neurotransmitter targets. J Psychopharmacol. 2018; 32: 96-104.

42. Krege S, Goepel M, Sperling H, Michel MC. Affinity of trazodone for human penile alpha1- andalpha2-adrenoceptors. BJU Int. 2000; 85: 959-61.

43. Choi Y, Park C, Jang J, Kim S, Jeon H, Kim W, et al. Effects of Korean ginseng berry extract on sexual function in men with erectile dysfunction: a multicenter, placebo-controlled, double-blind clinical study. Int J Impot Res. 2013; 25: 45-50.

44. Oh K-J, Chae M-J, Lee H-S, Hong H-D, Park K. Effects of Korean red ginseng on sexual arousal in menopausal women: placebo-controlled, double-blind crossover clinical study. J Sex Med. 2010; 7: 1469-77.

45. Murphy LL, LEE TJF. Ginseng, sex behavior, and nitric oxide. Ann N Y Acad Sci. 2002; 962: 372-7.

46. Kim S-o, Lee M, Xui Y, Ahn KY, Hong H-D, Kim SS, et al. Effects of Korean Red Ginseng on the Vaginal Blood Flow and Structure in Female Castrated Rats. Korean J Urol. 2006; 47: 888-94.

47. Kim SO, Kim MK, Lee HS, Park JK, Park K. The effect of Korean red ginseng extract on the relaxation response in isolated rabbit vaginal tissue and its mechanism. J Sex Med. 2008; 5: 2079-84.

48. Farnia V, Hojatitabar S, Shakeri J, Rezaei M, Yazdchi K, Bajoghli H, et al. Adjuvant Rosa damascena has a small effect on SSRI-induced sexual dysfunction in female patients suffering from MDD. Pharmacopsychiatry. 2015; 48: 156-63.

49. Farnia V, Shirzadifar M, Shakeri I Rezaei M, Bajoghli $\mathrm{H}$, Holsboer-Trachsler E et al. Rosa damascena oil improves SSRI-induced sexual dysfunction in male patients suffering from major depressive disorders: results from a double-blind, randomized, and placebo-controlled clinical trial. Neuropsychiatr Dis Treat. 2015; 11: 625

50. Jahromi HK, Jashni HK, Dialemeh S. Effect of Damask rose extract on FSH, LH and testosterone hormones in rats. Int J Med Res Health Sci. 2016; 5: 267-71. 